Würde die Verfassungsänderung vom Volk angenommen, würde sie Grundlage und wichtige Voraussetzung für weitere Forderungen bilden, wie sie beispielsweise von der Nationalen Ethikkommission (NEK) bereits formuliert sind [2]: Eizellspende, Embryonenspende, Leihmutterschaft, HLA-Typisierung (letztlich für Retterbabys).

Die Grafik im Artikel von B. Imthurn ist ungenau und soll den Eindruck erwecken, dass alleine die Schweiz und Litauen «rückständig» seien in ihren Gesetzgebungen zur Fortpflanzungsmedizin. In Deutschland z.B. ist die PID zwar zugelassen, aber nur «wenn Paare eine Veranlagung für eine schwerwiegende Erbkrankheit in sich tragen oder bei ihnen mit einer Tot- oder Fehlgeburt zu rechnen ist.» [3] Die vorliegende Verfassungsänderung würde zukünftig der Schweiz eines der liberalsten Fortpflanzungsmedizingesetze innerhalb Europas bescheren. Wozu?
Wir Bürger - und dazu gehören auch wir Ärztinnen und Ärzte - müssen die rechtlichen Konsequenzen mitbedenken: Gewisse Befürworter der Verfassungsänderung, vertreten von Markus Hofmann in der NZZ, hinterfragen bereits grundsätzlich die Pflicht des Rechtsstaates zum Schutz des Lebens. [4] Wo sind wir gelandet, wenn diese wichtigste Aufgabe des Staates hinterfragt wird?

1 Maio G. Mittelpunkt Mensch: Ethik in der Medizin. Stuttgart: Schattauer; 2012. S. 232.

2 Die medizinisch unterstützte Fortpflanzung. NEK Stellungnahme Nr. 22/2013.

3 www.bundestag.de/dokumente/textarchiv/2011/ 35036974_kw27_de_pid/205898

4 Dem moralischen Kompass der Bürger vertrauen, NZZ 24.3.2015. "Statt nur nach Gründen zu suchen, wieso die PID erlaubt werden soll, sollte man fragen, was dem Staat eigentlich das Recht verleiht, seinen moralisch mündigen Bürgern ein PID-Verbot vorzuschreiben."

\title{
Gute ethische Gründe für die PID
}

\section{Nikola Biller-Andorno}

Prof. Dr. med. Dr. phil., Institut für Biomedizinische Ethik und Medizingeschichte, Universität Zürich, Mitglied im Fachkomitee «Ja zur Fortpflanzungsmedizin»

Korrespondenz:

Prof. Dr. med. Dr. phil.

Nikola Biller-Andorno

Institut für Biomedizinische

Ethik und Medizin-

geschichte

Universität Zürich

Pestalozzistrasse 24

CH-8032 Zürich

biller-andorno[at]

ethik.uzh.ch
Es mag nahe liegen, die Befürworter von PID mehr auf der Seite medizinisch-technischer Möglichkeiten und die Skeptiker auf der Seite der Ethik zu vermuten. Dem ist aber nicht so: Zwar gibt es ohne Zweifel erwägenswerte ethische Einwände gegen die Präimplantationsdiagnostik, die diskutiert werden müssen. Diesen stehen jedoch gute moralische Gründe für die Zulassung der PID gegenüber - gerade unter den Rahmenbedingungen, wie sie heute in der Schweiz anzutreffen sind.

$\mathrm{Zu}$ diesen gehört die rege Nutzung der Pränataldiagnostik, die (beim Vorliegen bestimmter Voraussetzungen) von Fachgesellschaften empfohlen und von den Kassen übernommen wird. Dies führt bei einem positiven Befund zu einem Schwangerschaftskonflikt und häufig zur Abtreibung. Die PID bietet die Möglichkeit, diese Situation wenigstens in einem Teil der Fälle zu vermeiden. Auf diese Option zu verzichten, ist moralisch fragwürdig und bedürfte einer sorgfältigen Rechtfertigung.

Zwar werden auch bei der PID Embryonen verworfen, doch geschieht dies in einem sehr frühen Entwicklungsstadium, noch vor der Implantation - ein Zeitpunkt, bis zu dem auch im Laufe des natürlichen
Befruchtungsprozess ein signifikanter Anteil von Embryonen zugrunde geht.

Auch wäre es unfair, Paaren, die sich wegen Unfruchtbarkeit oder einer Erbkrankheit für eine In-Vitro-Fertilisation mit PID entscheiden, zu unterstellen, sie seien in selbstsüchtiger Manier am perfekten Nachwuchs interessiert. Nicht das "Aussondern von Embryonen mit «unerwünschtem» Erbgut» ist das Ziel, sondern das Vermeiden von Leid für das künftige gewünschte, vielfach heiss ersehnte - Kind sowie allenfalls die Erhöhung der Erfolgschancen einer körperlich und psychisch belastenden IVF-Behandlung. Die Gefahr der Eugenik, darin ist den Kritikern der assistierten Reproduktion Recht zu geben, ist nicht von der Hand zu weisen. Doch die Verirrungen eugenischen Denkens und sogar ihre Umsetzung sind letztlich unabhängig von technologischen Voraussetzungen. Eugenik beginnt im Kopf und nicht in der Petri-Schale.

Es braucht daher Wachsamkeit auf gesellschaftlicher Ebene, um entsprechende Tendenzen zu erkennen und gegensteuern zu können. Ärztinnen und Ärzte können hier einen entscheidenden Beitrag leisten. 